\title{
Rise in brittle fracture resistance of spherical vessels for storage of liquid ammonia under extreme forces
}

\author{
E. M. Basko, V. V. Larionov \& V. N. Lazutin \\ Melnikov institute of steel structures, Moscow, Russia
}

\begin{abstract}
This report presents the results of spherical vessel investigation and laboratory research of physico-mechanical properties of steel and welded joints. Dynamics of corrosion-mechanical cracking of the vessel welded joints and brittle fracture resistance at low temperatures and dynamic forces have been given in the context of fracture mechanics. The major causes of corrosion-mechanical cracking of the vessel welded joints in the medium of liquid ammonia have been determined. The performance of works on stress deconcentration in the butt-welded joints on the inside of the wall being in contact with ammonia was demonstrated to be an effective method of increasing resistance to corrosion-mechanical cracking and brittle fracture.
\end{abstract}

Keywords: spherical vessel, ammonia, pressure, cracks, defects, welded joints, life, cracking resistance, brittle fracture, dynamic stress.

\section{Introduction}

Practice of application of the welded pressure vessels for storage of liquid ammonia demonstrated that corrosion-mechanical cracking (CMC) of welded joints is made possible in the course of their service. The action of extreme forces in cracking can result in brittle fracture of vessels at stresses that are substantially below the design values. This is associated both with the resulting dynamic effects and with dependence of cracking resistance characteristics and critical temperatures of vessel material brittleness on strain rate. In this connection when evaluating resistance to fracture of vessels for storage of ammonia on action of extreme forces it is necessary to take into account 
damages accumulating during service, on the one hand, and minimize formation and growth of cracks during the design period of service by use of the suitable materials and technologies, on the other hand.

Russia first faced with a CMC problem for the spherical vessels with a volume of $2000 \mathrm{~m}^{3}$, in which storage of liquid ammonia is carried out at the excessive pressure of $0.6 \mathrm{MPa}$ and a temperature as high as $12^{\circ} \mathrm{C}$, in the middle $80 \mathrm{~s}$ when during their inspection cracking of the welded joints was discovered and several vessels failed ahead of schedule (Pohodnya et al. [1], Basko et al. [2]).

In this connection the institute accepted a program, on CMC-problem research which included execution of full-scale investigation of technical condition of liquid ammonia pressure vessels, study of physico-mechanical properties of vessel material and development of methods for increase of resistance to corrosion-mechanical cracking and brittle fracture taking into account the effect of low temperatures and dynamic effects.

The effect of low temperatures and dynamic loading are considered as the extreme cases exceeding the bounds of design conditions of vessel service. Decrease in temperature of wall to minus $30-40^{\circ} \mathrm{C}$ is made possible in case of break of wall thermal insulation and depressurization of wall and leakage of ammonia can result in its local supercooling up to minus $67^{\circ} \mathrm{C}$.

The dynamic loads are possible as a result of the external actions of seismic or wind character, et al. as well as initiation of a brittle crack in the zone of local supercooling of the wall section during its depressurization, action of which is comparable with the external dynamic loading by its effect (Basko et al. [3]).

\section{Analytical treatment of investigation results}

Since 1990 there was carried out an investigation of technical condition of 12 spherical vessels after a lapse of $6,8,12$ and 24 years of their operation. There was performed inspection of the base metal and welded joints on the inside of the wall being in contact with liquid and gaseous ammonia.

The visual inspection of the welded joints showed a great deal of technological defects in the form of gas pockets, chains of undercuts, flux inclusions, high ripple of weld beads, et al. practically in all vessels. The primary inspection of the welded joints by methods of color and ultrasonic test did not discover cracks.

To increase a sensitivity of capillary method on one of the first vessels, there was carried out the mechanical removal of the weld bead bulge with the subsequent grinding flush with the base metal. First of all such work was performed in the butt-welded joints with technological welding defects. Inspection of the welded joints prepared by the mentioned above way demonstrated a rich variety of the surface cracks. The crack width was equal to 6-10 microns and this fact required the careful preparation of the welded joints surfaces for their detection. In this connection later on inspection of the welded joints was carried out only after the mentioned mechanical preparation of welds, length of which in one spherical vessel amounts to $\approx 700 \mathrm{~m}$. 
Inspection of the welded joints with the following grinding of welds showed the surface cracks in all investigated vessels. All cracks were activated because of the welding defects. Length and number of cracks depended on type and size of the welding defects as well the vessel service life. The lowest number of cracks (20 pcs 3-15 mm in length) was discovered in the spherical vessel after 6 years of its service [2]. At the same time the welded joints of this vessel had the lowest number of technological defects allowable for welding. The welding cracks were oriented both along and across axis of welds. The length of cross cracks was no more than $50 \mathrm{~mm}$ and that of longitudinal cracks was as high as 2 $\mathrm{m}$ [2]. The depth of longitudinal cracks was no more than $8 \mathrm{~mm}$ whereas that of cross cracks reached $18 \mathrm{~mm}$. Cases of the wall depressurization associated with crack propagation were not registered. The typical distribution of cracks throughout the depth obtained on the basis of inspection results of 2 spherical vessels after 24 years of their service is presented in fig.1; in each of these vessels more than 270 cracks were discovered.

As evident from the cited data, more than $50 \%$ of the surface cracks were as deep as $2 \mathrm{~mm}$. These cracks were formed from small defects of welds up to 0.5 $\mathrm{mm}$ in depth. Only $5 \%$ of cracks had a depth of more than $2 \mathrm{~mm}$. The maximum depth of cracks reached a value of $17-18 \mathrm{~mm}$. At the same time the similar presence of defects is made also possible for a smaller service life, but with a larger quantity and sizes of technological defects. The analogous data on damageability of 4 spherical vessels after 16 years of their service are presented in the work of Kuzeev [4]; according to its information more than 160 surface cracks were discovered in each of these vessels.

The corrosion-mechanical cracking of welds in the liquid ammonia medium occurs in the cylindrical pressure vessels too (Basko et al. [2]). However in connection with a far less length of welds, on the one hand, and a lower level of stresses resulting from internal pressure and higher quality of welds, on the other hand, the total quantity of these cracks is significantly less than in the spherical vessels.

Following the primary inspection of the welded joints all discovered defects were eliminated by a mechanical way. The defective parts of the welded joints with a depth of crack sampling more than $3 \mathrm{~mm}$ were subjected to backing run, grinding flush with the base metal and inspection. After the hydraulic test operation all the investigated vessels were approved for their service.

In future there was monitoring of four investigated spherical vessels. In accordance with it the careful inspection of the welded joints for defect detection was carried out every 2-3 years of their service. As a result of repeatedly executed inspection it was determined that following the technological operation of stress deconcentration in the butt-welded joints the corrosion-mechanical cracking was practically arrested. The discovered individual cracks to $10-20 \mathrm{~mm}$ in length resulted from the small cracks missing during the primary inspection or those that were incompletely removed during the initial repair. The last investigation of spherical vessel that was carried out following 29 years of its service and 15 years after its primary inspection and repair confirmed efficiency of the executed technological operations providing considerable increase in 
resistance to corrosion-mechanical cracking of welds in the ammonia medium. Following investigation of mechanical properties of material cut from the wall of the given vessel and calculations of the service life and resistance to brittle fracture it was allowed to the further service for a period up to 2014 years.

\section{Research results of physico-mechanical properties of the spherical vessel material}

To evaluate effect of the long-term exposure of ammonia on the physicomechanical properties of 09G2S steel (spr Russian standard) and welded joints, there was carried out cut-out of the wall fragments from the spherical vessels after 8, 12 and 29 years of their service. The results of vessel material investigation were compared with the corresponding data for 09G2S steel sheets and welded joints.

The results of laboratory research of the chemical analysis, mechanical properties and microstructure of the vessel material showed that all characteristics satisfy the requirements of standards for base metal and welded joints (Basko et al. [2]). The similar results were obtained in the course of the vessel material investigation after 29 years of its service.

As follows from the results of experimental investigations, the values of critical stress intensity factors $K_{c}$ obtained by the results of vessel material tests are no less than the corresponding characteristics gained in testing samples made from sheets and butt-welded joints non-subjected to ammonia action. The successful welding of inserts into the walls of spherical vessels instead of cutout fragments confirmed preservation of the good metal weldability. This fact is an indirect evidence of steel hydrogenation absence in process of continuous service, as also agreed with the research results of hydrogen analysis of metal presented in the work of Basko et al. [2].

The performed laboratory research testifies the possible usage of mechanical properties and cracking resistance characteristics obtained in testing the spherical vessel material non-subjected to action of liquid ammonia for calculation of the load-carrying capacity of brittle fracture resistance of vessels aimed for storage of liquid ammonia.

\section{Design estimation of the spherical vessel service life}

As follows from the investigation results, formation of cracks in the welded joints of spherical vessels is possible already at the early stage of their operation. In process of propagation they can reach the values approaching thickness of the vessel wall. At the same time at the design stage the strength calculations are carried out without taking presence of cracks into consideration. This introduces uncertainty as into the actual load-carrying capacity of wall as in fixing the safe operation life of vessels.

On the basis of analysis of damageability dynamics for the welded joints of spherical vessels for 6-30 years of their service and performed investigations of mechanical properties and cracking resistance characteristics of vessel material 
we carried out a design estimation of resistance to corrosion cracking and brittle fracture with use of methods and criteria of fracture mechanics.

In accordance with points of the fracture mechanics time $T$ to formation of crack from the source defect $a_{o}$ to the calculated value $a_{p}$ can be represented in the form of eqn (1):

$$
T=T_{z}+T_{p}
$$

where $\quad T_{z} \quad$ - time to crack birth from a technological defect;

$T_{p} \quad$ - time of crack growth from $a_{o}$ to $a_{p}$.

Since, as the results of investigation indicated, time to the crack birth can be ranged from 0 to 5 years with existing technology of welding and quality of the welded joints, calculations of the service life assume to take $T_{z}=0$. Then the design service life is determined by time of crack growth from $a_{o}$ to the accepted design size $a_{p}$ and according to Karzov et al. [5] it can be calculated by integration of eqn (2).

$$
T_{p}=C_{\kappa} \int_{a_{o}}^{a_{p}} \Delta K^{-y} d a
$$

where $\Delta K \quad-$ difference of stress intensity factors;

$a_{o}$ and $a_{p}-$ initial and design sizes of crack;

$C_{\kappa} \quad-$ empirically determined coefficient characterizing resistance to corrosion-mechanical cracking in the liquid ammonia medium.

Determination of $C_{k}$ in laboratory conditions is a very intricate and laborintensive problem connected with assurance of test conditions approaching the actual tests over a long period of time. On the basis of damage dynamics analysis of spherical vessels made from 09G2S low-alloy steel and exploited at the operating pressure up to $0.6 \mathrm{MPa}$ we obtained $C_{\kappa}=(1.8-2.0) \cdot 10^{2} \mathrm{MPa} \sqrt{m}$. The empirically determined relationship for $T_{p}$ can be represented in the form of eqn (3):

$$
T_{p}=\frac{C_{\kappa}}{\sigma}\left[\frac{1}{M_{o} \sqrt{a_{o}}}-\frac{1}{M_{p} \sqrt{a_{o p}}}\right]
$$

where $\sigma \quad$ - design stresses in the wall of vessel from the operating pressure;

$M_{o}$ and $M_{p}$ - coefficients taking the relative sizes and form of crack into account.

Fig.1 presents the results of calculations according to eqn (3) with different sizes $a_{o}$ of source defects for the spherical vessels with a volume of $2000 \mathrm{~m}^{3}$ at the operating stress $\sigma=120 \mathrm{MPa}$. The measured values of the crack depth in the vessel welded joints are also presented here (marked by daggers). As follows from Fig.1, the design curves reflect the total kinetics mechanisms of the welded joint damageability for the spherical vessels. The discovered damageability to 
$(0.5-0.9) t$ at the early stage of service of $8-12$ years is concerned with presence of the source technological defects $1.5-2.5 \mathrm{~mm}$ in depth (curves 4-6). In the presence of the source defects $0.5-1.0 \mathrm{~mm}$ deep the similar damageability takes place after 20-25 years of service (curves 1-3). With defects less than $0.5 \mathrm{~mm}$ deep the depth of cracks for service up to 24 years does not exceed $0.2 t$ at $t=20$ $\mathrm{mm}$.

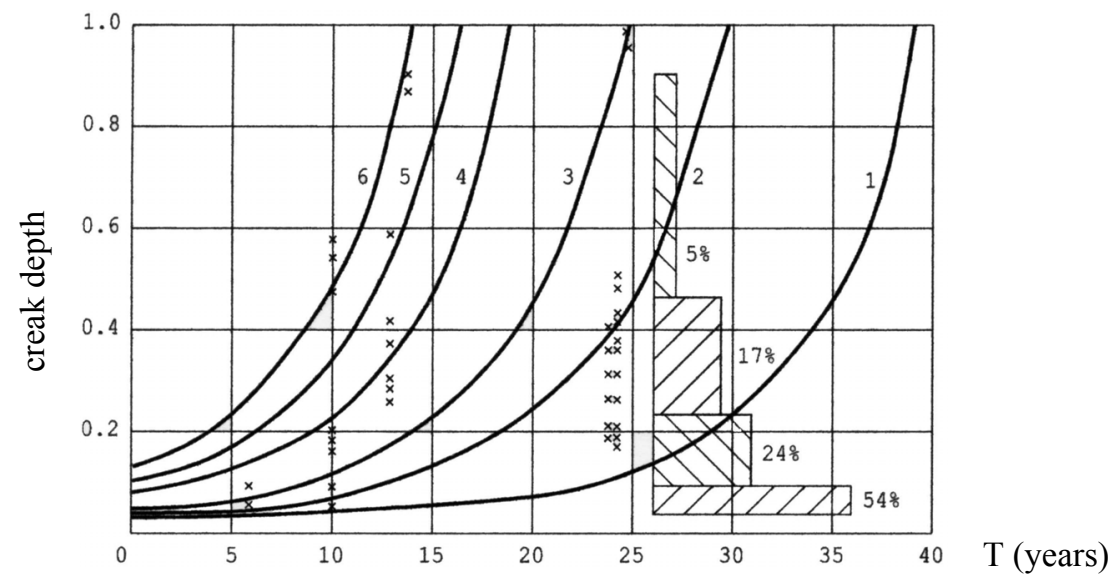

Figure 1: Diagram "Crack depth-time of service T (years)" (LZZD distribution of crack depth after 24 years of service).

To analyze resistance to brittle fracture and service life of spherical vessels taking size and type of defects, stress level, temperature and dynamic forces into consideration, it is more convenient to represent eqn (3) in the form of eqn (4):

$$
T_{p}=C_{\kappa} \frac{\Delta K}{K_{o}-K_{p}}
$$

where $\Delta K \quad$ - difference of stress intensity factors $K_{o}$ and $K_{p}$;

$K_{o}$ and $K_{p} \quad$ - initial and design values of stress intensity factors.

Formation of through-the-thickness crack results in depressurization of wall and so this fact eliminates possibility of the further service of vessel. Therefore accepting a safety factor as a value of $K_{p}$ corresponding to formation of throughthe-thickness crack and replacing $K_{p}$ by $\sigma \sqrt{\pi t}$ from eqn (4) we obtain an expression for a design service period of spherical vessel in the form of eqn (5):

$$
T_{p}=C_{\kappa}\left(\frac{1}{K_{o}}-\frac{n_{\kappa}}{\sigma \sqrt{\pi t}}\right)
$$

where $\quad n_{k}=2-$ safety factor;

$$
\begin{array}{ll}
\sigma & - \text { operating stress in the wall of vessel; } \\
t & - \text { thickness of wall. }
\end{array}
$$


Substituting the numerical values $K_{o}=4.0 \mathrm{MPa}$ (at $a_{o}=0.5 \mathrm{~mm}$ ), $\sigma=120 \mathrm{MPa}$, $t=20 \mathrm{~mm}, n_{\kappa}=2$ and $C_{\kappa}=180$ into the eqn (5) we obtain that the design service life is equal to 33 years according to criterion of brittle fracture resistance at $a_{o} \leq$ $0.5 \mathrm{~mm}$.

As it follows from the eqn (6), increase in CMC resistance may be reached by decrease in size $a_{o}$ of the source defects, reduction of operating stresses $\sigma$ by means of the operating pressure reduction or increase in thickness of the vessel wall.

\section{Evaluation of brittle fracture resistance}

As mentioned above, decrease in temperature of the vessel wall is possible as a result of break of the vessel thermal insulation or depressurization of wall. In the last case temperature of wall on the local section of depressurization zone can fall to minus $67^{\circ} \mathrm{C}$. The results of tension test of samples with through-thethickness cracks showed that under the static loading transition of samples to brittle fracture at stresses that are less than yield strength takes place at temperatures below minus $80^{\circ} \mathrm{C}$. At a temperature of minus $70^{\circ} \mathrm{C}$ the critical stress intensity factor $K_{c}$ is no less than $85 \mathrm{MPa} \sqrt{m}$ (Basko et al. [2]). With respect to the value of $K_{p}=30 \mathrm{MPa} \sqrt{m}$ corresponding to formation of throughthe-thickness crack the safety factor $n_{k}$ as to the critical value of $K_{c}$ is no less than 2.8 and with respect to the allowable value of $\left[K_{p}\right]$ it is more than 5.0. This is evidence of the adequate supplies of load-carrying capacity of vessels as to criterion of brittle fracture resistance at the static loading.

The action of dynamic loads caused by the external and internal factors noted in introduction results in increase in the strain rate of material in the crack tip. In this case the critical temperatures of transition from tough to quasibrittle and brittle fractures can essentially rise. The feature of low-alloy steel behavior under conditions of dynamic load action lies in sensitivity of their mechanical properties and cracking resistance characteristics to the strain rate. At the same time the influence of strain rate becomes apparent in different ways depending on capability for plastic deformation. In case of preservation of capability for plastic deformation the yield strength of low-alloy steel increases. The value of critical stress intensity factor $K_{C} \dot{\varepsilon}$ is calculated by eqn (6) (Basko [6]):

$$
K_{C} \dot{\varepsilon}=K_{C} \dot{\varepsilon}_{0} \frac{\sigma_{T \dot{\varepsilon}}}{\sigma_{T \dot{\varepsilon}_{0}}}
$$

where $K_{C} \dot{\mathcal{\varepsilon}}$ and $K_{C} \dot{\mathcal{E}}_{0}$ - values of $K_{C}$ at the strain rates $\dot{\varepsilon}$ and $\dot{\varepsilon}_{0}$.

During dynamic loading the strain rate can reach the value of $10^{2}-10^{3} \mathrm{~s}^{-1}$. The yield strength of low-alloy steels at the given strain rates increases by $1.5-1.7$ times. The cracking resistance limit of steel under dynamic loading increases too Basko and Makhutov [7]. Therefore in the field of tough and quasitough conditions under action of dynamic loads the brittle fracture resistance is provided. 
As follows from the results of impact resistance test of samples and double tension test of large-size flat samples with initiation of brittle cracks (Basko et al. [3]), the critical temperature of transition from quasitough to quasibrittle fractures for $09 \mathrm{G} 2 \mathrm{~S}$ steel under dynamic loading is equal to minus $10-20^{\circ} \mathrm{C}$. Therefore at the temperatures of vessel wall below minus $10^{\circ} \mathrm{C}$ the evaluation of resistance to brittle fracture taking dynamic forces into account is to be carried out with use of dynamic characteristics of cracking resistance.

According to Basko and Makhutov [7] characteristics of dynamic cracking resistance of $09 \mathrm{G} 2 \mathrm{~S}$ steel are $K_{d}=20 \mathrm{MPa} \sqrt{m}$ and $30 \mathrm{MPa} \sqrt{m}$ at temperatures of minus $70^{\circ} \mathrm{C}$ and minus $40^{\circ} \mathrm{C}$ respectively. At the given values of dynamic cracking resistance limit $K_{d}$ the brittle fracture resistance of vessels under dynamic forces is provided prior to their depressurization at the wall temperatures above minus $40^{\circ} \mathrm{C}$. In case of wall depressurization and decrease of temperature to minus $67^{\circ} \mathrm{C}$ on the local section of zone of through-thethickness crack formation the crack arrest is possible at the wall temperature outside the supercooled section above minus $10^{\circ} \mathrm{C}$ on condition that length of brittle crack is expressed by eqn (7) (Basko and Makhutov [7]):

$$
2 l_{d} \leq \frac{2}{\pi}\left(\frac{K_{d}}{\sigma \cdot n_{\kappa}}\right)
$$

where $2 l_{d} \quad$ - length of brittle crack;

$K_{d} \quad$ - dynamic limit of cracking resistance;

$\sigma \quad-$ stress in the wall of vessel;

$n=1,2-$ safety factor as to the dynamic cracking resistance limit.

Substituting the corresponding values into eqn (7) we obtain the crack length $2 l_{d}$ $<110 \mathrm{~mm}$ at which the brittle fracture resistance of spherical vessel is provided in case of local depressurization of wall.

\section{Conclusion}

The results of performed investigations are evidence of practical expediency and applicability of the fracture mechanics concepts both for dynamics analysis of corrosion-mechanical cracking, evaluation of brittle fracture resistance of the steel vessels taking a stressed state, quality of manufacturing and service conditions into account and analysis of extreme cases associated with action of low temperatures and dynamic loading. The obtained results of investigations made it possible to formulate the basic positions on calculation of service life and evaluation of brittle fracture resistance of the spherical vessels as well as to propose an effective method of increase in resistance to corrosion-metallic cracking of the welded joints in the liquid ammonia medium.

At the same time it is necessary to perform the further investigations for more accurate definition of CMC resistance characteristics of steels, effect of residual welding stresses and thermal treatment on the process of initiation and 
propagation of cracks as well as execution of investigations to develop a welding technology providing a high uniformity of mechanical properties of butt-welded joints, first of all during realization of extreme actions and conditions.

\section{References}

[1] Pokhodnya I.K., Lebedev B.F. et al. Features of crack formation in the welded vessels for storage of liquid ammonia in the process of their service. Automatic Welding, 10, pp. 39-42, 1988.

[2] Basko E.M., Demygin N.E., Goncharova Y.E. Increase in safe operation life of vessels for storage of liquid ammonia under pressure. Factory Laboratory, 2, pp. 51-58, 1997.

[3] Basko E, Larionov V., Lazutin V. Influence of dynamic effects on the resistance of steel building structures to brittle failures. WIT Transactions on the Built Environment, 87, pp. 127-136, 2006.

[4] Kuzeev R.D. Experience of technical diagnostics (examination of industrial safety) of the spherical vessels with a volume of $2000 \mathrm{~m}^{3}$ for storage of liquid ammonia at Mendeleevskazot Company Ltd., Republic of Tatarstan. Research News. Publication of Close Corporation "INOKhIM", 2-3, pp. 42-45, 2006.

[5] Karzov G.P., Leonov V.P., Timofeev B.T. Welded pressure vessels. "Mashinostroyenie", Leningrad, p. 287, 1982.

[6] Basko E.M. Cracking resistance of structural steels and construction units. Proceeding Book of TsNIIproektstalkostruktsiya. Research of brittle strength of the metal building structures, pp.112-128, 1982.

[7] Basko E.M., Makhutov N.A. Research of cracking resistance of structural steels under dynamic initiation and propagation of brittle cracks. Problems of fracture and safety of technical systems. Collection of Scientific Proceeding, Krasnoyarsk, pp. 44-49, 1997. 